\title{
Complexity Analysis of Resting-State fMRI in Adult Patients with Attention Deficit Hyperactivity Disorder: Brain Entropy
}

\author{
Gülsüim Akdeniz \\ Faculty of Medicine, Department of Biophysics and Yenimahalle Training and Research Hospital, \\ Ankara Yıldirım Beyazıt University, Ankara, Turkey \\ Correspondence should be addressed to Gülsüm Akdeniz; gakdeniz@ybu.edu.tr
}

Received 6 July 2017; Revised 25 October 2017; Accepted 21 November 2017; Published 12 December 2017

Academic Editor: Silvia Conforto

Copyright (C) 2017 Gülsüm Akdeniz. This is an open access article distributed under the Creative Commons Attribution License, which permits unrestricted use, distribution, and reproduction in any medium, provided the original work is properly cited.

Objective. Complexity analysis of functional brain structure data represents a new multidisciplinary approach to examining complex, living structures. I aimed to construct a connectivity map of visual brain activities using resting-state functional magnetic resonance imaging (fMRI) data and to characterize the level of complexity of functional brain activity using these connectivity data. Methods. A total of 25 healthy controls and 20 patients with attention deficit hyperactivity disorder (ADHD) participated. fMRI preprocessing analysis was performed that included head motion correction, temporal filtering, and spatial smoothing process. Brain entropy (BEN) was calculated using the Shannon entropy equation. Results. My findings demonstrated that patients exhibited reduced brain complexity in visual brain areas compared to controls. The mean entropy value of the ADHD group was $0.56 \pm 0.14$, compared to $0.64 \pm 0.11$ in the control group. Conclusion. My study adds an important novel result to the growing literature pertaining to abnormal visual processing in ADHD that my ADHD patients had lower BEN values, indicating moreregular functional brain structure and abnormal visual information processing.

\section{Introduction}

Functional magnetic resonance imaging (fMRI) is among the most powerful tools for noninvasive assessment of behavior, cognition, and psychiatric disorders [1] and is used to obtain volumetric images, including time-resolution data pertaining to human brain function. In particular, the resting-state [2] fMRI technique is a preferred, alternative tool to assess brain function abnormalities in psychiatric disorders. Bloodoxygen-level-dependent signals of resting-state fMRI allow for the analysis of functional connectivity patterns within brain networks [3] and the temporal dynamics of activity fluctuations therein [4].

The most complex living structure known to man is the human brain. The reason that complexity is assessed using resting-state fMRI, with respect to psychiatric diseases, is that complex output patterns in a living system can indicate its health and robustness [5]. Complex living systems, such as the human brain, develop to possess maximum adaptive capacity [6]. The deterioration of, and reductions in, the essential functions of these complex systems, in accordance with aging and disease, is associated with a loss of complexity in the dynamics of complex physiological systems [7]. Chaotic and complex behaviors are indicative of a healthy system, whereas more predictable and regular behaviors can denote pathological states [8].

Attention deficit hyperactivity disorder (ADHD) is a common neurodevelopmental disorder that typically begins in childhood, often persists into adulthood, and is associated with consistent deficits in error processing and inhibition and regionally decreased grey matter volume $[9,10]$. Although diagnosis is made on a behavioral basis, cognitive deficits may also be significant, especially in terms of executive function [11, 12] and attentional processes $[13,14]$. Numerous neuroimaging studies have been conducted on ADHD; Bush $[5,15]$ reviewed several functional imaging studies and observed a consistent pattern of frontal dysfunction in ADHD patients. However, few studies have examined both frontal and other brain regions [16].

Entropy, a powerful indicator of irregularity in a system [17], is not associated with the value of a random variable, but depends only on the distribution of values. Entropy can 
characterize the level of chaos in, and complexity of, a system, within the context of information theory [18]. In medical image processing applications, entropy provides a measure of the heterogeneity of the distribution of data in the image matrix. When all data are identical, the entropy value is zero; this value increases commensurately with differences in the data and its distribution. Entropy is a useful tool in neuroscience research for obtaining meaningful results from the analysis of fMRI data [19-21]. In previous fMRI studies, entropy was considered first as an innovative, alterative indicator [22] and then as a means of detecting activation [1]; it is viewed currently as a potential marker of brain diseases $[23,24]$. Measurements of brain entropy (BEN) may be used to make inferences regarding brain status and alterations due to disease [21].

The aim of the present study was (i) to determine the complexity of visual brain activity by calculating brain entropy and (ii) to assess differences between attention deficit hyperactivity disorder patients and healthy controls in terms of brain status.

\section{Material and Methods}

2.1. Resting-State fMRI Data Acquisition. The fMRI images used in this study were downloaded freely from the website of the 1000 Functional Connectomes Project [25]. The gender distribution in the ADHD group $(n=25)$ was as follows: 20 males and 5 females, ranging in age between 20 and 50 years. The following scanning parameters were used: $\mathrm{TR}=2$; \# slices = 39; and \# timepoints = 192. The second group, comprising healthy volunteers $(n=20)$, included 8 males and 12 females between 18 and 46 years of age; in this group, the following scanning parameters were applied: $\mathrm{TR}=2$; \# slices $=33$; and \# timepoints $=175$.

All research conducted by ADHD-200 contributing sites was conducted with local IRB (institutional review board) approval and contributed in compliance with local IRB protocols. All data distributed via the International Neuroimaging Data-Sharing Initiative is fully anonymized in compliance with HIPAA (The Health Insurance Portability and Accountability Act) Privacy Rules.

2.2. Image Analysis. FEAT fMRI preprocessing analysis of the downloaded 4D fMRI data sets was performed using the FSL [26] software package. Standard preprocessing analysis includes head motion correction, temporal filtering, and spatial smoothing process. All data were filtered according to a high-pass filter cut-off value of $100 \mathrm{~s}$, motion-corrected, and smoothed with a Gaussian kernel using a full width at half maximum value of $5 \mathrm{~mm}$. Independent component analysis (ICA) is a computational technique for revealing hidden factors that underlie fMRI raw data. In this study, the MELODIC (Multivariate Exploratory Linear Optimized Decomposition into Independent Components) independent component analysis (ICA) technique was used to separate single fMRI data sets into different spatial and temporal components in analyzing the fMRI data. Each subject's structural image was registered to standard Montreal Neurological Institute space (i.e., to the MNI152 template) for the purposes of spatial normalization. As a result of the analysis, components 17-44 were produced for each patient.

2.3. Entropy Calculation. Various components for each participant, including one pertaining to the most meaningful pattern of visual activation, were selected to assess complexity (one participant was excluded from the study, because no meaningful visual activation could was detected). The brain entropy values of the two sequential images most closely matched and, with the minimum degree of noise, were calculated for each subject using a program written using the MATLAB GUI (Mathworks, Inc.) software package [27]. This program was used to evaluate the brain entropy mapping from fMRI data. Entropy was calculated using Shannon's entropy as follows:

$$
\mathrm{BEN}=-\sum_{i=1}^{256} p(i) \log _{2} p(i),
$$

where $p(i)$ is the normalized probability function of the pixel intensity $i$ of the image.

Shannon entropy is a measure of how much information is required, on average in a given discrete probability distribution. The probability function of the pixel intensities was acquired and normalized by dividing by the total pixel number.

Directly calculating BEN from fMRI data is challenging and prone to inaccuracy due to the effects of the image pixels that represent the brain tissue and to their negative correlation with entropy values. To overcome this, I segmented the images selected for BEN analysis according to their color. Profiles of five important colors were given in color image segmentation process: the background color, white, grey, blue, and red (Figure 1(a)). A display of these segmentation techniques and their application to extract white, grey, and blue colors is shown in Figure 1(b). After extraction, the remaining red color signals which do not represent the activation of occipital lobe had to be considered as an artifact and removed from the images in Figure 1(c). Figure 1 provides a flowchart of the color image processing steps necessary to calculate BEN.

2.4. Statistical Analysis. Parametric statistical analysis was performed using the Statistical Package for Social Sciences (SPSS16.0; Chicago, IL, USA) software. $t$-test was conducted for differences between the mean entropy values in the ADHD group and control group. Mann-Whitney $U$ test was performed to reveal the group differences between the patient and control groups in gender and age. The relationship between the BEN and group and between gender and group was determined by using Pearson's chi-squared test.

\section{Results}

I have achieved fMRI components and Figure 2 depicts two example fMRI components. It should be noted that I selected and used, during calculation of BEN, only components exhibiting meaningful visual activation, such as those shown below. 


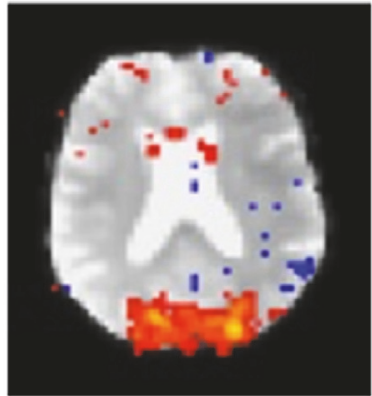

(a)

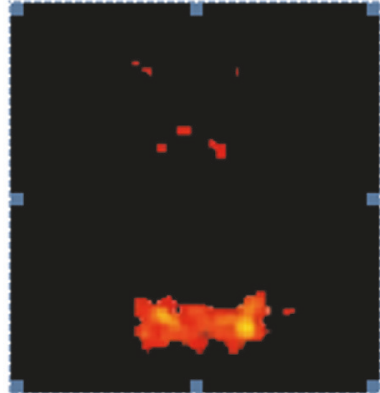

(b)

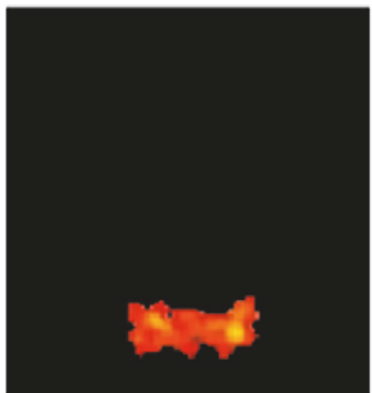

(c)

FIGURE 1: Flowchart of the color image segmentation process used to extract visual activation data: (a) fMRI image, (b) color segmentation process, and (c) a visual activation data.

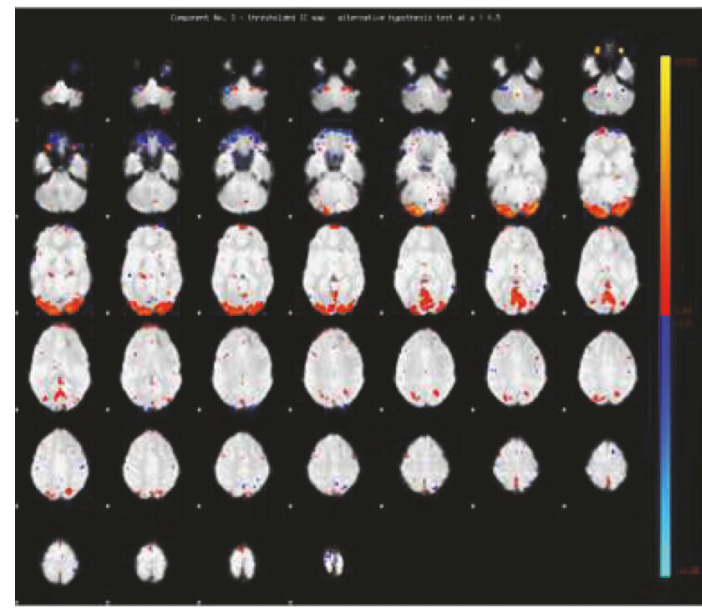

(a)

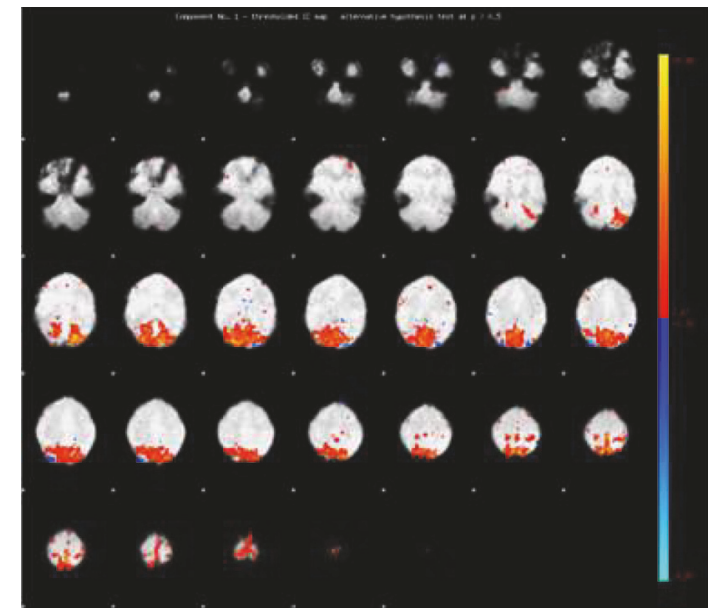

(b)

FIGURE 2: Images produced by fMRI analysis denoting visual activation in (a) an ADHD patient and (b) a control participant.

The BEN bars of the ADHD patients and controls are depicted in Figure 3. The mean entropy value of the ADHD group was $0.56 \pm 0.14$, compared to $0.64 \pm 0.11$ in the control group; this difference was significant $(p=0.008)$.

Table 1 lists the comparisons results between the patient and control groups. There were significant group differences in gender $(p=0.010)$ and age $(p=0.045)$. Data are provided as means $\pm \mathrm{SD}$.

On correlation analysis, there was a negative relationship between BEN and group $(r=-0.306 ; p=0.043)$ and between gender and group $(r=-0.390 ; p=0.009)$.

\section{Discussion}

Throughout the past decade, the majority of studies on psychiatric diseases have been fMRI-based investigations [10, 28, 29]. I studied ADHD using this technique due to the importance of functional connectivity in psychiatric diseases and because of the ability of fMRI to index this connectivity and activity within the brain. I employed BEN mapping, of functional brain connectivity, to better understand the disease.
Complexity analysis of functional brain structure [30] is a promising tool with which to examine functional brain connectivity at an organizational level [31]. The degree of complexity is associated with the number of brain connections; decreased connectivity indicates lower complexity, and increased connectivity reflects greater complexity. Numerous studies on brain activity in ADHD have been performed using functional neuroimaging techniques other than fMRI, such as electroencephalography and magnetoencephalography [32, 33]. Gómez et al. [32] demonstrated that MEG recordings of ADHD patients were more-regular compared to recordings obtained in a control group; furthermore, there were significant differences among these groups in five brain regions, that is, anterior, central, posterior, left lateral, and right lateral areas. Sokunbi et al. [34] used restingstate fMRI to demonstrate reduced complexity in the brain activity of adult ADHD patients compared to healthy, agematched controls. van den Heuvel and Hulshoff Pol [3] suggested resting-state fMRI studies examining functional connectivity have provided a new and promising platform to examine hypothesized disconnectivity effects in psychiatric brain diseases. There has been a reawakening of interest in an alternative approach that focuses on the resting state [15]. I 
TABLE 1: Comparison of group characteristics.

\begin{tabular}{|c|c|c|c|c|}
\hline & $\operatorname{ADHD}(n=25)$ & Control $(n=20)$ & $Z / \chi^{2}$ & $p$ \\
\hline Age & $34.52 \pm 9.54$ & $29.32 \pm 10.02$ & -2.007 & 0.045 \\
\hline Gender* (male/female) & $20 / 5$ & $8 / 11$ & 6.699 & 0.010 \\
\hline Brain entropy (BEN) & $0.56 \pm 0.14$ & $0.64 \pm 0.11$ & -2.666 & 0.008 \\
\hline \multicolumn{5}{|l|}{ ADHD-RS } \\
\hline Inattentive & $16.79 \pm 4.90$ & - & - & - \\
\hline Hyperactive/impulsive & $13.13 \pm 5.17$ & - & - & - \\
\hline Total & $29.92 \pm 8.75$ & - & - & - \\
\hline \multicolumn{5}{|l|}{ ACDS } \\
\hline Inattentive & $6.59 \pm 2.52$ & - & - & - \\
\hline Hyperactive/impulsive & $7.82 \pm 1.33$ & - & - & - \\
\hline
\end{tabular}

Mann-Whitney $U$ test, ${ }^{*}$ Pearson's chi-squared test; ADHD-RS: ADHD-Rating Scale; ACDS: Adult ADHD Clinical Diagnostic Scale.

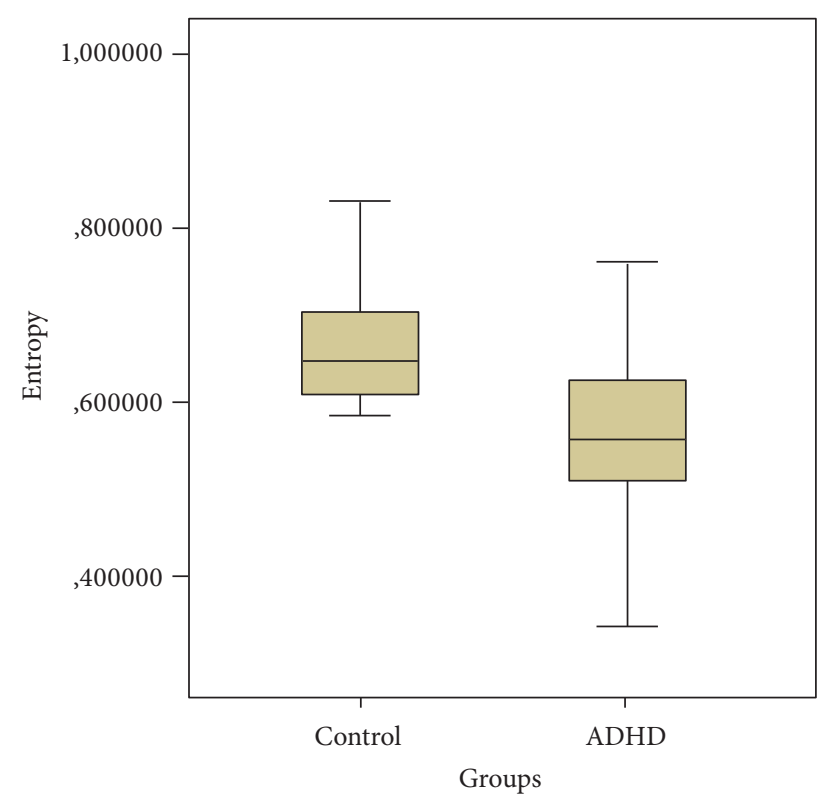

FIGURE 3: BEN bars of the ADHD patient and control groups.

investigated functional brain connectivity in occipital areas using resting-state fMRI; in contrast to previous studies, I observed a more-regular pattern of complexity, in the context of visual activities, in the patient group compared to the control group. A previous structural study reported decreased total brain and occipital lobe volume in ADHD patients [35, 36]. I suggested that this reduction in occipital lobe volume may account for the reduced functional brain connectivity, and more-regular pattern of complexity, observed in ADHD.

BEN can quantify the complexity of the functional architecture of the human brain [30], such that the BEN values I obtained during my complexity analysis provide a physiologically and functionally meaningful account of the brain activity of both ADHD patients and controls. My BEN results, obtained from fMRI image analyses, also show that individuals with ADHD exhibit lower entropy in particular brain regions compared to controls, thereby indicating a more-regular pattern of complexity. This is consistent with Goldberger and Lipsitz's model of robustness [5, 7, 37-39] in which the complexity of a system's physiological output decreases commensurately with greater age and disease. Therefore, I proposed that entropy may represent a useful indicator for research on various brain states.

Several researchers have reported abnormal frontalstriatal brain function in patients with ADHD [40]. However, a growing number of studies also indicate abnormal posterior brain function, and associated abnormalities, in early-stage sensory information processing [41]. According to a recent meta-analysis of fMRI studies examining task-based cognition in ADHD, functional abnormalities in the visual cortex may represent a key finding in ADHD [41]. My study adds an important novel result to the growing literature pertaining to abnormal visual processing in ADHD; that is, my ADHD patients had lower BEN values, indicating more-regular functional brain structure and abnormal visual information processing. Furthermore, I demonstrated abnormalities in brain function in the occipital region using resting-state $\mathrm{fMRI}$. Abnormal visual information processing in ADHD has been also identified by previous studies, consistent with my results $[42,43]$.

Numerous studies indicated that ADHD patients exhibit gender differences in clinical and sociodemographic characteristics [44-46]. Taken together, the results of these studies are in general agreement in terms of suggesting that young female ADHD patients exhibit lower ratings on hyperactivity, inattention, impulsivity, and externalizing problems, in addition to greater intellectual impairments and internalizing of problems, compared to young males with ADHD [37]. The prevalence of ADHD in the adult population is $4.4 \%$ in the US, of whom $38 \%$ are female and $62 \%$ are male (National Resource Center on ADHD). These data are generally supported by my study, with respect to gender differences between the study groups, but not in terms of BEN values. These results support Rubin and colleagues' study; they suggested that fMRI applications of complexity have dealt with both between the voxel and between-subject differences [30].

My BEN results indicate that excessive orderliness is not advantageous and in fact indicates abnormal function; greater complexity indicates a healthier system. I believe that using entropy analyses during fMRI may be of benefit to research 
in various pathologic and nonpathologic areas. Furthermore, I suggest that BEN can index brain activity and may help to determine abnormalities in brain activity, for example, in pilot studies of drug addiction [47].

In conclusion, the present study successfully demonstrated a reduction in BEN in ADHD patients compared to healthy controls, by calculating signal entropy (Shannon entropy) in accordance with the level of complexity of resting brain activity. This result supports the notion that the complexity of resting brain activity can be used as an indicator of ADHD.

\section{Conflicts of Interest}

The author declares that they have no conflicts of interest.

\section{References}

[1] D. B. De Araujo, W. Tedeschi, A. C. Santos, J. Elias Jr., U. P. C. Neves, and O. Baffa, "Shannon entropy applied to the analysis of event-related fMRI time series," NeuroImage, vol. 20, no. 1, pp. 311-317, 2003.

[2] M. E. Raichle, A. M. MacLeod, A. Z. Snyder, W. J. Powers, D. A. Gusnard, and G. L. Shulman, "A default mode of brain function," Proceedings of the National Acadamy of Sciences of the United States of America, vol. 98, no. 2, pp. 676-682, 2001.

[3] M. P. van den Heuvel and H. E. Hulshoff Pol, "Exploring the brain network: a review on resting-state fMRI functional connectivity," European Neuropsychopharmacology, vol. 20, no. 8, pp. 519-534, 2010.

[4] A. Rotarska-Jagiela, V. Vande Ven, V. Oertel-Knöchel, P. J. Uhlhaas, K. Vogeley, and D. E. J. Linden, "Resting-state functional network correlates of psychotic symptoms in schizophrenia," Schizophrenia Research, vol. 117, no. 1, pp. 21-30, 2010.

[5] G. Bush, "Neuroimaging of attention deficit hyperactivity disorder: can new imaging findings be integrated in clinical practice?", Child and Adolescent Psychiatric Clinics of North America, vol. 17, no. 2, pp. 385-404, 2008.

[6] C. Wolf and D. E. J. Linden, "Biological pathways to adaptability - interactions between genome, epigenome, nervous system and environment for adaptive behavior," Genes, Brain and Behavior, vol. 11, no. 1, pp. 3-28, 2012.

[7] L. A. Lipsitz, "Physiological complexity, aging, and the path to frailty," Science of aging knowledge environment: SAGE KE, vol. 2004, no. 16, pe16 pages, 2004.

[8] R. Pool, "Is it healthy to be chaotic?" Science (New York, NY), vol. 243, no. 4891, pp. 604-607, 1989.

[9] A. Mary, H. Slama, P. Mousty et al., "Executive and attentional contributions to Theory of Mind deficit in attention deficit/hyperactivity disorder (ADHD)," Child Neuropsychology, vol. 22, no. 3, pp. 345-365, 2016.

[10] R. Iannaccone, T. U. Hauser, J. Ball, D. Brandeis, S. Walitza, and S. Brem, "Classifying adolescent attention-deficit/hyperactivity disorder (ADHD) based on functional and structural imaging," European Child and Adolescent Psychiatry, vol. 24, no. 10, pp. 1279-1289, 2015.

[11] J. A. Sergeant, H. Geurts, and J. Oosterlaan, "How specific is a deficit of executive functioning for attention-deficit/hyperactivity disorder?" Behavioural Brain Research, vol. 130, no. 1-2, pp. $3-28,2002$.
[12] E. G. Willcutt, A. E. Doyle, J. T. Nigg, S. V. Faraone, and B. F. Pennington, "Validity of the executive function theory of attention-deficit/ hyperactivity disorder: a meta-analytic review," Biological Psychiatry, vol. 57, no. 11, pp. 1336-1346, 2005.

[13] O. Tucha, S. Walitza, L. Mecklinger et al., "Attentional functioning in children with ADHD - Predominantly hyperactiveimpulsive type and children with ADHD - Combined type," Journal of Neural Transmission, vol. 113, no. 12, pp. 1943-1953, 2006.

[14] A. Pasini, C. Paloscia, R. Alessandrelli, M. C. Porfirio, and P. Curatolo, "Attention and executive functions profile in drug naive ADHD subtypes," Brain \& Development, vol. 29, no. 7, pp. 400-408, 2007.

[15] Y. Paloyelis, M. A. Mehta, J. Kuntsi, and P. Asherson, "Functional MRI in ADHD: A systematic literature review," Expert Review of Neurotherapeutics, vol. 7, no. 10, pp. 1337-1356, 2007.

[16] J. R. Sato, D. Y. Takahashi, M. Q. Hoexter, K. B. Massirer, and A. Fujita, "Measuring network's entropy in ADHD: A new approach to investigate neuropsychiatric disorders," NeuroImage, vol. 77, pp. 44-51, 2013.

[17] S. I. Sandler, Chemical, Biochemical, and Engineering Thermodynamics, Wiely, New York, NY, USA, 2006.

[18] C. E. Shannon, "A mathematical theory of communication," ACM SIGMOBILE Mobile Computing and Communications Review, vol. 5, no. 1, pp. 3-55, 2001.

[19] E. Tavazzi, M. G. Dwyer, B. Weinstock-Guttman et al., "Quantitative diffusion weighted imaging measures in patients with multiple sclerosis," NeuroImage, vol. 36, no. 3, pp. 746-754, 2007.

[20] J.-L. Cui, C.-Y. Wen, Y. Hu, T.-H. Li, and K. D.-K. Luk, "Entropybased analysis for diffusion anisotropy mapping of healthy and myelopathic spinal cord," NeuroImage, vol. 54, no. 3, pp. 21252131, 2011.

[21] G. Akdeniz, Z. Erdogan, İ. Atli, and M. İ. Atagün, "Complexity of the brain activity in the patients with schizophrenia using resting-state fMRI," in Proceedings of the In 5th World Congress of Asian Psychiatry, Fukuoka , Japan, 2015.

[22] R. Baumgartner, R. Somorjai, R. Summers, W. Richter, and L. Ryner, "Novelty indices: Identifiers of potentially interesting time-courses in functional MRI data," Magnetic Resonance Imaging, vol. 18, no. 7, pp. 845-850, 2000.

[23] D. S. Bassett, B. G. Nelson, B. A. Mueller, J. Camchong, and K. O. Lim, "Altered resting state complexity in schizophrenia," NeuroImage, vol. 59, no. 3, pp. 2196-2207, 2012.

[24] A. C. Yang, C.-C. Huang, H.-L. Yeh et al., "Complexity of spontaneous BOLD activity in default mode network is correlated with cognitive function in normal male elderly: A multiscale entropy analysis," Neurobiology of Aging, vol. 34, no. 2, pp. 428438, 2013.

[25] NITRC, Functional Connectomes Project, 2015, http://www .nitrc.org/projects/fcon_1000/.

[26] Analysis Group FMRIB, Oxford, FMRIB Software Library, 2015, https://fsl.fmrib.ox.ac.uk/fsl/fslwiki/.

[27] The MathWorks, Inc. MathWorks, 2015, http://www.mathworks .com/.

[28] W. Gao, Q. Jiao, S. Lu et al., "Alterations of regional homogeneity in pediatric bipolar depression: A resting-state fMRI study," BMC Psychiatry, vol. 14, no. 1, article no. 222, 2014.

[29] J. M. Ford, V. A. Palzes, B. J. Roach et al., "Visual hallucinations are associated with hyperconnectivity between the amygdala and visual cortex in people with a diagnosis of schizophrenia," Schizophrenia Bulletin, vol. 41, no. 1, pp. 223-232, 2015. 
[30] D. Rubin, T. Fekete, and L. R. Mujica-Parodi, "Optimizing complexity measures for FMRI data: algorithm, artifact, and sensitivity," PLoS ONE, vol. 8, no. 5, Article ID e63448, 2013.

[31] M. Rubinov and O. Sporns, "Complex network measures of brain connectivity: Uses and interpretations," NeuroImage, vol. 52, no. 3, pp. 1059-1069, 2010.

[32] C. Gómez, J. Poza, M. García, A. Fernandez, and R. Hornero, "Regularity analysis of spontaneous MEG activity in AttentionDeficit/ Hyperactivity Disorder," in Proceedings of the 33rd Annual International Conference of the IEEE Engineering in Medicine and Biology Society, EMBS 2011, pp. 1765-1768, USA, September 2011.

[33] A. Cerquera, M. Arns, E. Buitrago, R. Gutierrez, and J. Freund, "Nonlinear dynamics measures applied to EEG recordings of patients with Attention Deficit/Hyperactivity Disorder: Quantifying the effects of a neurofeedback treatment," in Proceedings of the 34th Annual International Conference of the IEEE Engineering in Medicine and Biology Society, EMBS 2012, pp. 1057-1060, USA, September 2012.

[34] M. O. Sokunbi, W. Fung, V. Sawlani, S. Choppin, D. E. J. Linden, and J. Thome, "Resting state fMRI entropy probes complexity of brain activity in adults with ADHD," Psychiatry Research: Neuroimaging, vol. 214, no. 3, pp. 341-348, 2013.

[35] F. X. Castellanos, J. N. Giedd, P. C. Berquin et al., "Quantitative brain magnetic resonance imaging in girls with attentiondeficit/hyperactivity disorder," Archives of General Psychiatry, vol. 58, no. 3, pp. 289-295, 2001.

[36] F. Xavier Castellanos, P. P. Lee, W. Sharp et al., "Developmental trajectories of brain volume abnormalities in children and adolescents with attention-deficit/hyperactivity disorder," Journal of the American Medical Association, vol. 288, no. 14, pp. 17401748, 2002.

[37] A. L. Goldberger, "Non-linear dynamics for clinicians: chaos theory, fractals, and complexity at the bedside," The Lancet, vol. 347, no. 9011, pp. 1312-1314, 1996.

[38] A. L. Goldberger, "Fractal variability versus pathologic periodicity: Complexity loss and stereotypy in disease," Perspectives in Biology and Medicine, vol. 40, no. 4, pp. 543-561, 1997.

[39] A. L. Goldberger, L. A. N. Amaral, J. M. Hausdorff, P. C. Ivanov, C.-K. Peng, and H. E. Stanley, "Fractal dynamics in physiology: alterations with disease and aging," Proceedings of the National Acadamy of Sciences of the United States of America, vol. 99, no. 1, pp. 2466-2472, 2002.

[40] S. Durston, J. van Belle, and P. de Zeeuw, "Differentiating frontostriatal and fronto-cerebellar circuits in attentiondeficit/hyperactivity disorder," Biological Psychiatry, vol. 69, no. 12, pp. 1178-1184, 2011.

[41] S. Cortese, C. Kelly, C. Chabernaud et al., "Toward systems neuroscience of ADHD: a meta-analysis of 55 fMRI studies," The American Journal of Psychiatry, vol. 169, no. 10, pp. 10381055, 2012.

[42] J. Wang, T. Jiang, Q. Cao, and Y. Wang, "Characterizing anatomic differences in boys with attention-deficit/hyperactivity disorder with the use of deformation-based morphometry," American Journal of Neuroradiology, vol. 28, no. 3, pp. 543-547, 2007.

[43] T. Sigi Hale, A. M. Kane, O. Kaminsky et al., "Visual network asymmetry and default mode network function in ADHD: An fMRI study," Frontiers in Psychiatry, vol. 5, article no. 81, 2014.

[44] J. Gershon, "A meta-analytic review of gender differences in ADHD," Journal of Attention Disorders, vol. 5, no. 3, pp. 143-154, 2002.
[45] F. Levy, D. A. Hay, K. S. Bennett, and M. McStephen, "Gender differences in ADHD subtype comorbidity," Journal of the American Academy of Child and Adolescent Psychiatry, vol. 44, no. 4, pp. 368-376, 2005.

[46] U. P. Ramtekkar, A. M. Reiersen, A. A. Todorov, and R. D. Todd, "Sex and age differences in attention-deficit/hyperactivity disorder symptoms and diagnoses: implications for DSM-V and ICD-11," Journal of the American Academy of Child and Adolescent Psychiatry, vol. 49, no. 3, pp. 217-228, 2010.

[47] J. S. ZW, Y. Li, Z. Singer, R. Ehrman, A. V. Hole, C. P. O’Brien et al., "Human brain entropy mapping using thousands of subjects and its application in a drug addiction study," in Proceedings of the Annual Meeting of Society for Neuroscience, 7491 pages, San Diego, Calif, USA, 2013. 

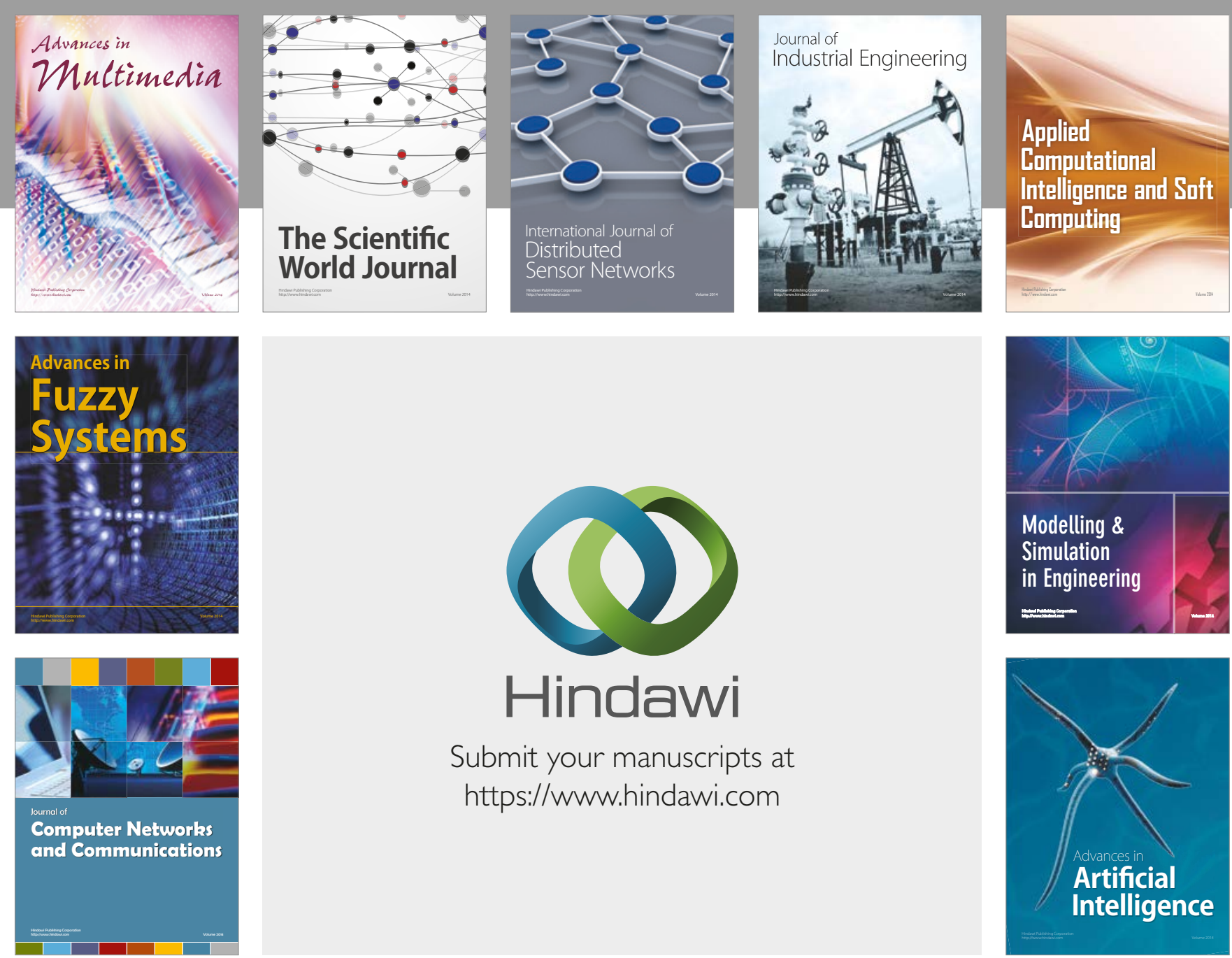

\section{Hindawi}

Submit your manuscripts at

https://www.hindawi.com
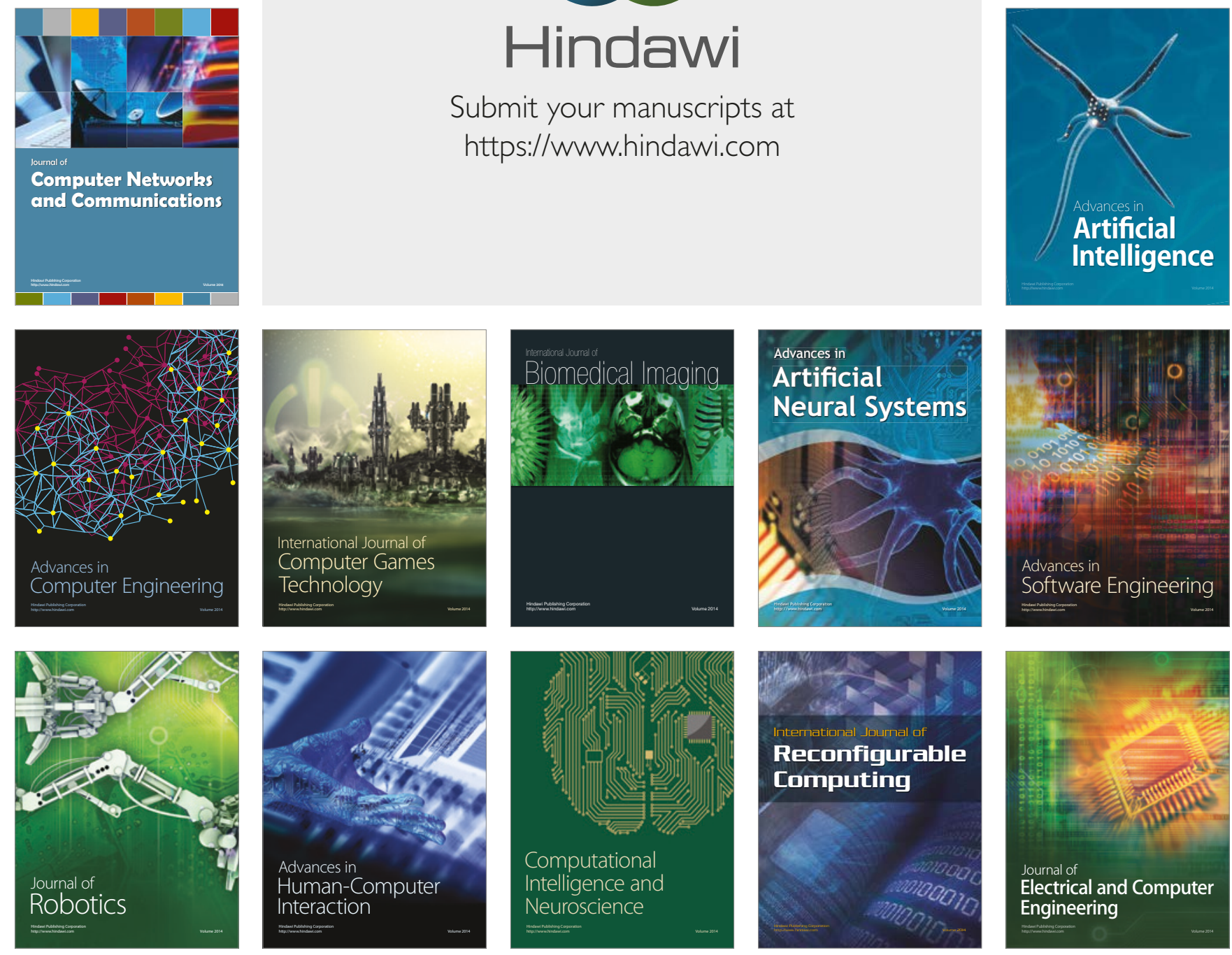